\title{
Numerical solutions for a nearly circular crack with developing cusps under shear loading.
}

\begin{abstract}
In this paper, we study the behavior of the solution at the crack edges for a nearly circular crack with developing cusps subject to shear loading. The problem of finding the resulting force can be written in the form of a hypersingular integral equation. The equation is then transformed into a similar equation over a circular region using conformal mapping. The equation is solved numerically for the unknown coefficients, which will later be used in finding the stress intensity factors. The sliding and tearing mode stress intensity factors are evaluated for cracks and displayed graphically. Our results seem to agree with the existing asymptotic solution.
\end{abstract}

Keyword: Nearly circular crack; Numerical method; Galerkin method; Stress intensity factors; Hypersingular integral equation; Shear loading; Conformal mapping. 